\title{
The effect of lamotrigine augmentation of clozapine in a sample of treatment-resistant schizophrenic patients: A double-blind, placebo-controlled study
}

\author{
R. Zoccali ${ }^{\text {a,* }}$, M.R. Muscatello ${ }^{\text {a }}$, A. Bruno ${ }^{a}$, R. Cambria ${ }^{a}$, \\ U. Micò $^{\mathrm{a}}$, E. Spina ${ }^{\mathrm{b}}$, M. Meduri ${ }^{\mathrm{a}}$ \\ ${ }^{a}$ Section of Psychiatry, Department of Neurosciences, Psychiatric and Anaesthesiological Sciences, University of Messina, Italy \\ ${ }^{\mathrm{b}}$ Section of Pharmacology, Department of Clinical and Experimental Medicine and Pharmacology, \\ University of Messina and IRCCS Centro Neurolesi "Bonino-Pulejo”, Messina, Italy
}

\begin{abstract}
Based on the evidence that lamotrigine added to clozapine in refractory schizophrenic patients has reported promising results, the present 24-week double-blind, randomized, placebo-controlled trial had the aim to explore the efficacy of lamotrigine add-on pharmacotherapy on clinical symptomatology and cognitive functioning in a sample of treatment-resistant schizophrenic patients receiving clozapine. After clinical and neurocognitive assessments patients were randomly allocated to receive, in a double-blind design, either up to $200 \mathrm{mg}$ /day of lamotrigine or a placebo. A final sample of fifty-one patients completed the study.

The results obtained indicate that lamotrigine added to stable clozapine treatment showed a beneficial effect on the negative, positive and general psychopathological symptomatology in a sample of treatment-resistant schizophrenic patients. Regarding cognitive functions, improvement was observed in some explored areas, such as attentional resistance to interference, verbal fluency and executive functioning. The findings provide evidence that lamotrigine augmentation of clozapine treatment is well tolerated and may be proposed as an effective therapeutic strategy to improve outcome in treatment-resistant schizophrenia.
\end{abstract}

Keywords: Lamotrigine; Schizophrenia; Augmentation; Clozapine; Treatment; Resistant

\section{Introduction}

The treatment of schizophrenia, a severe and chronic brain disease, still remains a challenge. Despite the

\footnotetext{
* Corresponding author. Department of Neurosciences, Psychiatric and Anesthesiological Sciences, Policlinico Universitario Via Consolare Valeria-Messina 98125 Italy. Tel.: +39 090 22212092; fax: +39 090695136.

E-mail address: Rocco.Zoccali@unime.it (R. Zoccali).
}

introduction of atypical antipsychotics, whose mechanism of action has been attributed to the antagonism of 5-HT2A receptors coupled to weaker antagonism of dopamine D2 receptors, only $10-20 \%$ of schizophrenic patients show a good outcome, recovering to pre-illness levels of functioning, while another $15-20 \%$ show a poor outcome and are considered treatment-resistant; the middle group exhibits a range of residual cognitive, psychotic, affective and psychosocial symptoms despite medications (Tamminga and Holcomb, 2005). 
Dysfunction of glutamatergic transmission is thought to play a significant role in the pathophysiology of schizophrenia, representing a potential target for therapeutic strategies (Tamminga, 1999; Goff and Coyle, 2001). Two opposing hypotheses on glutamate involvement in schizophrenia have been proposed: the glutamate hypofunction hypothesis and the glutamate hyperfunction hypothesis. The first hypothesis has received support from the observation that $N$-methylD-aspartate (NMDA) receptor antagonists, such as the dissociative anesthetics phencyclidine (PCP) and ketamine can induce an amotivational state, psychotic symptoms, and disrupted processing of sensory information in healthy volunteers (Halberstadt, 1995), as well as exacerbating psychotic symptoms and cognitive impairment in schizophrenic patients (Jentsch and Roth, 1999; Haroutunian et al., 2003). Reduced concentrations of glutamate in the cerebrospinal fluid of schizophrenic patients have been reported (Kim et al., 1980), and abnormalities in glutamatergic receptors in the cortex and temporal lobe have emerged from postmortem studies (Ishimaru et al., 1994).

The glutamate hyperfunction hypothesis was proposed on the basis of the evidence of the excess of glutamatergic synapses in the frontal cortex of schizophrenic patients (Simpson et al., 1998). Additional evidence has derived from studies that have found an increase in the activity of phosphate activated glutaminase, the glutamate synthesizing enzyme (Gluck et al., 2002).

Even if apparently conflicting, the hypo- and hyperfunction theories share a common finding, as the psychotomimetic effect of the dissociative anesthetics discussed before seems to be a result of increased glutamate release onto other receptors, as suggested by Anand et al. (2000) who have found that lamotrigine pre-treatment leads to attenuation of ketamine-induced neuropsychiatric effects.

Lamotrigine, an anticonvulsant drug, stabilizes presynaptic neuronal membranes and reduces excessive glutamate release in the brain via inhibition of voltagegated sodium and calcium channels (Kalyoncu et al., 2005). As noted by Kremer et al. (2004), the mechanisms of action of lamotrigine may be relevant to pharmacological treatment of schizophrenia, because of the large amount of evidence indicating the dysfunctional glutamatergic transmission in the pathophysiology of schizophrenia.

Previous studies with lamotrigine added to clozapine in refractory schizophrenic patients have reported promising results, such as the significant BPRS score reduction in partially responding or treatment-resistant schizophrenic patients on clozapine (Saba et al., 2002; Dursun et al., 1999). In a naturalistic follow-up case series (Dursun and Deakin, 2001), lamotrigine added to ongoing antipsychotic treatments at doses ranging from 100 to $300 \mathrm{mg}$ /day improved BPRS total scores only in clozapine-treated patients. More recently, a randomized, placebo-controlled study (Tiihonen et al., 2003) on 34 forensic schizophrenic inpatients on clozapine showed that the addition of $200 \mathrm{mg} /$ day of lamotrigine improved general psychopathology and positive symptoms. The efficacy of lamotrigine in addition to clozapine as well as other antipsychotics has been explored in a randomized, double-blind, placebo-controlled trial by Kremer et al. (2004) whose results demonstrated the efficacy of adjunctive lamotrigine up to $400 \mathrm{mg}$ /day on general psychopathology and residual positive symptoms. The potential role of lamotrigine in schizophrenia has also been reviewed by Large et al. (2005) who, examining clinical studies of adjunctive lamotrigine, suggested a combination of anti-dopaminergic and anti-glutamatergic drugs for the treatment of schizophrenia. An openlabel study of long-term adjunctive lamotrigine to antipsychotic monotherapy in 10 schizophrenic outpatients (Kolivakis et al., 2004) showed that the addition of lamotrigine at the mean dose of $232.5 \mathrm{mg}$ day produced overall benefit in patients with schizophrenia, as measured by changes on Clinical Global Impression (CGI) and PANSS scores. Results from five recent case studies (Thomas et al., 2006) showed that low dose lamotrigine (mean dose $=95 \mathrm{mg} /$ day) was a beneficial adjunctive treatment to a variety of antipsychotics, including conventional neuroleptics. Contrarily, a case report showed that the daily dose of $25 \mathrm{mg}$ lamotrigine added to quetiapine worsened psychotic symptoms (Chan et al., 2005).

Data on the potential effect of adjunctive lamotrigine on cognitive functioning in treatment-resistant schizophrenic patients are still lacking; a case report of an adolescent-onset schizophrenia treated with olanzapine and $75 \mathrm{mg}$ /day lamotrigine (aimed at preventing potential seizures) has shown the normalization of deficits in executive functions and verbal memory without a corresponding improvement in clinical symptomatology (Holzer and Halfon, 2004). A recent review of five relevant trials on lamotrigine for schizophrenia (Premkumar and Pick, 2006) has shown that the addition of lamotrigine may have a positive effect on the symptoms of schizophrenia, while it did not seem to have beneficial effects on cognitive functions; the authors concluded that more studies with larger samples are needed to confirm the real magnitude of efficacy and safety. 
Based on evidence from the literature, the present study has the aim of exploring the efficacy of lamotrigine add-on pharmacotherapy on clinical symptomatology and cognitive functioning in a sample of treatmentresistant schizophrenic patients receiving clozapine.

\section{Methods}

\subsection{Subjects}

The study was carried out at the Psychiatry Unit of the University Hospital of Messina, Italy.

Sixty outpatients, 34 males and 26 females, aged 2353 years old, who met DSM-IV criteria for schizophrenia and demonstrated persistent positive and negative symptoms despite an adequate trial of clozapine, were included in this study. Patients scoring 25 or more on the Brief Psychiatric Rating Scale (Overall and Gorham, 1962) at the baseline evaluation were classified as partial-responders or non-responders to clozapine (Munro et al., 2004). The patients' age, gender and duration of illness are shown in Table 1. The lamotrigine and placebo groups were compared for the different variables. All patients had been on clozapine monotherapy at the highest tolerable range $(150-650 \mathrm{mg} /$ day), for at least 1 year; the dose had been stable for at least 1 month prior to the study and was left unchanged throughout the study. The patients did not receive any antidepressant or anticonvulsant drugs for a period of 2 months prior to the study. During the study, patients were allowed to take lorazepam up to $5 \mathrm{mg} /$ day for insomnia or agitation. Patients with any other major psychiatric disorder, significant concurrent medical illnesses, organic brain disorder, history of substance and alcohol abuse, mental retardation, and pregnant or lactating women were excluded. All the patients provided written informed consent after a full explanation of the protocol design which had been approved by

Table 1

Demographic and clinical characteristics of the two groups (lamotrigine versus placebo)

\begin{tabular}{llll}
\hline & Lamotrigine & Placebo & $\mathrm{P}^{\mathrm{a}}$ \\
\hline Patients entered & 30 & 30 & - \\
Patients evaluable & 26 & 25 & - \\
Sex (M/F) & $15 / 11$ & $13 / 12$ & - \\
Age (years), mean \pm SD & $32.5 \pm 6.9$ & $30.2 \pm 7.8$ & 0.133 \\
$\begin{array}{l}\text { Duration of illness } \\
\quad \text { (years), mean } \pm \text { SD }\end{array}$ & $9.3 \pm 3.3$ & $10.4 \pm 4.3$ & 0.601 \\
$\begin{array}{l}\text { Clozapine dose } \\
\quad(m g / \text { day), mean } \pm \text { SD }\end{array}$ & $300 \pm 128.1$ & $335 \pm 128.5$ & 0.225 \\
\hline
\end{tabular}

a Mann-Whitney U-test. the local ethics committee. The patients were recruited from February 2005, and the follow-up was completed by September 2006.

\subsection{Study design}

This trial was a 24-week double-blind, randomized, placebo-controlled trial of adjunctive lamotrigine to clozapine therapy in schizophrenia. After baseline evaluation, subjects were randomly assigned to receive adjunctive treatment with either lamotrigine or placebo under double-blind conditions, using a randomization automated system on a 1:1 basis. During the study, the randomization list was held securely, and released only after study completion. Lamotrigine and the placebo were dispensed in identical-appearing capsules; patients randomized to placebo took the same number of capsules as those assigned to lamotrigine.

The dose of lamotrigine was increased from $25 \mathrm{mg} /$ day to $100 \mathrm{mg} /$ day at week 4, in increments of $25 \mathrm{mg} /$ week. The dose was maintained at $100 \mathrm{mg}$ /day for 8 weeks until week 12 . The dose was afterwards increased by a further $25 \mathrm{mg} /$ week until a dosage of $200 \mathrm{mg} /$ day was reached at week 16. This dosage was maintained until the end of the trial at week 24 . The rationale for this unique biphasic lamotrigine titration scheme was chosen to test the hypothesis that the efficacy of adjunctive lamotrigine might be dosedependent; the maximum dose of $200 \mathrm{mg}$ per day was established according to Tiihonen et al. (2003).

Clozapine and lamotrigine plasma level assays were used to test the patients' compliance to treatment and to verify possible drug interactions.

The following rating scales were used: the Brief Psychiatric Rating Scale (BPRS) (Overall and Gorham, 1962), the Scale for the Assessment of Negative Symptoms (SANS _ (Andreasen, 1983), the Scale for the Assessment of Positive Symptoms (SAPS) (Andreasen, 1984), and the Calgary Depression Scale for Schizophrenia (CDSS) (Addington et al., 1993). While inter-rater reliability for these assessments was not established by formal training, it is important to note that the assessments were conducted by psychiatrists with at least 5 years of clinical experience who were wellversed with the use of the rating scales.

Neurocognitive functioning was assessed with the Wisconsin Card Sorting Test (WCST) (Heaton et al., 1993), the verbal fluency task (Controlled Oral Word Association Test, Spreen and Benton, 1977), and the Stroop Colour-word Test (Trenerry et al., 1989). WCST is a commonly used measure of concept formation and flexibility of abstract thought in schizophrenia, though it 
is useful to assess executive functioning. Measures of performance included the number of categories completed and the number of perseverative errors. The tests were selected for the inclusion of functions frequently attributed to the frontal lobes and widely used in the study of cognition in schizophrenia.

Patients attended ten visits: initial screening (week -1 ), randomisation (week 0 ) and eight further visits at weeks 2, 4, 8, 12, 14, 16, 20, and 24. Data for clinical and neurocognitive assessments were collected at weeks 0, 12, and 24. Data for adverse events and EPS symptoms (elicited by non-specific questioning) were collected at each visit. The patients were also requested to report immediately any onset of possible rash or other skin reactions.

In addition to a physical examination, systolic and diastolic blood pressure, heart rate and body weight were all measured at each assessment. A routine set of laboratory investigations (blood profile, PT, aPTT, fibrinogen, basal glucose, cholesterol, triglycerids, uric acid, azotemy, AST, ALT, alkaline phosphatase, total and direct bilirubine, GGT, iron, ESR) was performed on all patients on admission and at the end of the study.

\subsection{Statistical analysis}

Data obtained from completers underwent check and quality control and, subsequently, descriptive and inferential statistical analysis. No last observation carried forward was performed. Comparison between the groups at baseline and at end of week 24 was performed using the Mann-Whitney test for two independent samples. The within-group differences in efficacy ratings between baseline and final test were analysed by the Wilcoxon rank sum test. The significance level for the test was $p<0.05$. Additionally, a Bonferroni correction was performed for the number of tests in each group. The statistical analysis was performed with Statistical Package for the Social Sciences (SPSS) 11.5 software (SPSS Inc, Chicago, IL, USA).

\section{Results}

Fifty-one patients completed the study and were included in the analyses of efficacy. There were nine premature dropouts, five in the placebo group and four in the lamotrigine group. Of the placebo group, three dropouts were due to concurrent illness, and two due to non-compliance with the visits. Of the lamotrigine group, three dropouts were due to non-compliance, and one due to the development of a rash.

At the baseline visit (day 0), there was no significant difference between active and control group for SANS, SAPS, BPRS, CDSS, Stroop test, verbal fluency, and WCST scores (Tables 2 and 3).

Table 2 shows the baseline and final values of the different efficacy variables for the lamotrigine and the placebo groups. Negative, positive, affective and overall clinical symptomatology improved significantly over the time of treatment (from baseline to week 24) in the lamotrigine group, but not in the placebo group. When the Bonferroni correction was performed for the number

Table 2

Clinical changes in patients receiving lamotrigine versus placebo at baseline and week 24

\begin{tabular}{|c|c|c|c|c|c|c|c|c|}
\hline & \multicolumn{2}{|l|}{ Lamotrigine } & \multicolumn{2}{|l|}{ Placebo } & \multicolumn{4}{|c|}{ Mann-Whitney $U$-test } \\
\hline & Baseline & Week 24 & Baseline & Week 24 & $\begin{array}{l}\text { Differ } \\
\text { baselin }\end{array}$ & & Differ & at week 24 \\
\hline \multicolumn{9}{|l|}{ SANS } \\
\hline Affective flattening & $14.9 \pm 6.8$ & $7.5 \pm 6.7$ & $14.4 \pm 7.3$ & $14.8 \pm 6.7$ & 318.5 & 0.901 & 120 & $<0.0001$ \\
\hline Alogia & $10.1 \pm 4.7$ & $3.9 \pm 3.3$ & $9.9 \pm 7.1$ & $9.8 \pm 6.9$ & 294 & 0.557 & 161 & $0.001 *$ \\
\hline Avolition/apathy & $7 \pm 4.9$ & $1.1 \pm 2.1$ & $7.1 \pm 4.3$ & $6.8 \pm 4.4$ & 316.5 & 0.872 & 157.5 & $0.001 *$ \\
\hline Anhedonia/Asociality & $10.5 \pm 7.2$ & $4.4 \pm 5.5$ & $10.6 \pm 6.2$ & $10.4 \pm 5.9$ & 304.5 & 0.697 & 152.5 & $0.001^{*}$ \\
\hline Attention & $4.7 \pm 4.8$ & $0.9 \pm 1.7$ & $4.7 \pm 3.6$ & $5.0 \pm 3.4$ & 311 & 0.787 & 113 & $<0.0001^{*}$ \\
\hline Total score & $47.5 \pm 25.3$ & $17.9 \pm 16.3$ & $47.3 \pm 24$ & $47.5 \pm 22.9$ & 322 & 0.955 & 76 & $<0.0001 *$ \\
\hline \multicolumn{9}{|l|}{$S A P S$} \\
\hline Hallucinations & $2.2 \pm 2.9$ & $0.2 \pm 0.6$ & $2.9 \pm 4.4$ & $2.8 \pm 4.2$ & 317.5 & 0.877 & 191.5 & $0.002^{*}$ \\
\hline Delusions & $5.8 \pm 7.3$ & $1.8 \pm 2.5$ & $5.5 \pm 4.9$ & $5.7 \pm 5$ & 301.5 & 0.649 & 191.5 & 0.010 \\
\hline Bizarre behaviour & $1 \pm 2.7$ & $0 \pm 0$ & $1.0 \pm 1.5$ & $1.0 \pm 1.5$ & 279 & 0.243 & 195 & $<0.0001^{*}$ \\
\hline Thought disorders & $4.5 \pm 6.4$ & $2 \pm 2.7$ & $4.4 \pm 4$ & $4.5 \pm 4$ & 260 & 0.207 & 185.5 & 0.007 \\
\hline Total score & $13.5 \pm 18$ & $4.1 \pm 3.5$ & $13.8 \pm 12.2$ & $14.0 \pm 12.2$ & 294 & 0.555 & 198 & 0.016 \\
\hline BPRS total score & $32.6 \pm 6.1$ & $24 \pm 4.4$ & $30.2 \pm 11.3$ & $31.5 \pm 6.7$ & 234 & 0.085 & 109 & $<0.0001^{*}$ \\
\hline CDSS total score & $5.1 \pm 4.8$ & $2.5 \pm 3.7$ & $5.6 \pm 4.3$ & $5.0 \pm 4.3$ & 288 & 0.481 & 174.5 & 0.004 \\
\hline
\end{tabular}

* Statistical significance after Bonferroni correction $(\alpha=0.002)$. 
Table 3

Cognitive functions at baseline (t0) and at week 24 in patients receiving lamotrigine versus placebo

\begin{tabular}{|c|c|c|c|c|c|c|c|c|}
\hline \multirow[b]{3}{*}{ Stroop test } & \multicolumn{2}{|c|}{ Lamotrigine } & \multicolumn{2}{|l|}{ Placebo } & \multicolumn{4}{|c|}{ Mann-Whitney $U$-test } \\
\hline & \multirow{2}{*}{$\begin{array}{l}\text { Baseline } \\
33.3 \pm 21\end{array}$} & \multirow{2}{*}{$\begin{array}{l}\text { Week } 24 \\
26.2 \pm 14.1\end{array}$} & \multirow{2}{*}{$\begin{array}{l}\text { Baseline } \\
41 \pm 28.7\end{array}$} & \multirow{2}{*}{$\begin{array}{l}\text { Week } 24 \\
42.3 \pm 25\end{array}$} & \multicolumn{2}{|c|}{$\begin{array}{l}\text { Difference at } \\
\text { baseline }\end{array}$} & \multicolumn{2}{|c|}{$\begin{array}{l}\text { Difference at week } \\
24\end{array}$} \\
\hline & & & & & 282 & 0.417 & 219.5 & 0.046 \\
\hline Phonemic fluency & $28.7 \pm 7.3$ & $30.4 \pm 8.7$ & $28.3 \pm 18.6$ & $22.3 \pm 10.9$ & 278 & 0.374 & 175 & 0.005 \\
\hline Semantic fluency & $39.1 \pm 10.7$ & $41.9 \pm 7.3$ & $36.2 \pm 10.8$ & $33.7 \pm 9.8$ & 263 & 0.241 & 156 & $0.001 *$ \\
\hline \multicolumn{9}{|l|}{ WCST } \\
\hline Perseverative errors & $33.5 \pm 23.3$ & $30.2 \pm 16$ & $32 \pm 28.2$ & $37.2 \pm 29.2$ & 308 & 0.748 & 292 & 0.533 \\
\hline$T$ scores perseverative & $39.4 \pm 17.8$ & $40 \pm 16.9$ & $36.3 \pm 12.9$ & $43.2 \pm 17.4$ & 317 & 0.875 & 281.5 & 0.402 \\
\hline Categories & $1.9 \pm 2.1$ & $2.5 \pm 1.9$ & $2 \pm 2.7$ & $2.2 \pm 2.6$ & 288 & 0.452 & 240 & 0.102 \\
\hline
\end{tabular}

* Statistical significance after Bonferroni correction $(\alpha=0.002)$.

of tests in each group, SANS domains and BPRS total score did not change; regarding SAPS scales, only the following factors remained statistically significant: hallucinations and bizarre behaviour.

The assessment of cognitive functioning of the patients receiving lamotrigine versus placebo showed significant changes in Stroop test and verbal fluency scores at week 24 (Table 3). After the Bonferroni correction was performed, only semantic fluency remained significant.

As shown in Table 4, lamotrigine augmentation of clozapine significantly reduced negative symptoms, and overall clinical symptomatology, as evidenced by changes on SANS and BPRS scores at week 12 (T1) and at the end of the trial; with regard to positive symptoms, improvement was seen in the scores for hallucinations, thought disorders and for total scores. Affective symptoms showed significant improvement only at week 24 , as documented by the reduction on CDSS scores. After the Bonferroni correction was performed, SANS domains, with the exception of attention, remained significant, while, among SAPS scales, only hallucinations reached statistical significance at week 12. Changes at CDSS were not confirmed and, regarding BPRS, only total score at week 24 remained significant.

With regard to cognitive functioning, as measured by Stroop test, verbal fluency and WCST, lamotrigine augmentation of clozapine significantly improved attentional resistance to interference, as assessed by Stroop test, and the task "categories" of WCST at week 24 , while the improvement in verbal fluency and in the

Table 4

Clinical changes in patients receiving lamotrigine at baseline, at week 12 (T1), and at week 24 (T2)

\begin{tabular}{|c|c|c|c|c|c|c|c|}
\hline \multirow[b]{2}{*}{ SANS } & \multirow[t]{2}{*}{ Baseline (T0) } & \multirow[t]{2}{*}{ Week 12 (T1) } & \multirow[t]{2}{*}{ Week 24 (T2) } & \multicolumn{2}{|c|}{ Difference $^{\mathrm{a}} \mathrm{T} 0$ vs T1 } & \multicolumn{2}{|c|}{ Difference $^{\mathrm{a}} \mathrm{T} 0$ vs $\mathrm{T} 2$} \\
\hline & & & & & & & \\
\hline Affective flattening & $14.9 \pm 6.8$ & $8.2 \pm 6.6$ & $7.5 \pm 6.7$ & -4.025 & $<0.0001 *$ & -3.065 & $0.002^{*}$ \\
\hline Alogia & $10.1 \pm 4.7$ & $4.3 \pm 5$ & $3.9 \pm 3.3$ & -4.029 & $<0.0001^{*}$ & -3.521 & $<0.0001^{*}$ \\
\hline Avolition/Apathy & $7 \pm 4.9$ & $3.2 \pm 5.2$ & $1.1 \pm 2.1$ & -3.641 & $<0.0001^{*}$ & -3.470 & $0.001 *$ \\
\hline Anhedonia/Asociality & $10.5 \pm 7.2$ & $5.7 \pm 6.5$ & $4.4 \pm 5.5$ & -3.717 & $<0.0001^{*}$ & -3.373 & $0.001 *$ \\
\hline Attention & $4.7 \pm 4.8$ & $3.1 \pm 4$ & $0.9 \pm 1.7$ & -2.571 & 0.010 & -2.864 & 0.004 \\
\hline Total score & $47.5 \pm 25.3$ & $26 \pm 27$ & $17.9 \pm 16.3$ & -4.310 & $<0.0001^{*}$ & -3.929 & $<0.0001 *$ \\
\hline \multicolumn{8}{|l|}{ SAPS } \\
\hline Hallucinations & $2.2 \pm 2.9$ & $0.6 \pm 1.4$ & $0.2 \pm 0.6$ & -3.035 & $0.002 *$ & -2.934 & 0.003 \\
\hline Delusions & $5.8 \pm 7.3$ & $3.5 \pm 4.7$ & $1.8 \pm 2.5$ & -1.777 & 0.076 & -2.456 & 0.014 \\
\hline Bizarre behaviour & $1 \pm 2.7$ & $0 \pm 0$ & $0 \pm 0$ & -1.857 & 0.063 & -1.857 & 0.063 \\
\hline Thought disorders & $4.5 \pm 6.4$ & $1.5 \pm 2.8$ & $2 \pm 2.7$ & -2.682 & 0.007 & -2.234 & 0.025 \\
\hline Total score & $13.5 \pm 18$ & $5.6 \pm 7.2$ & $4.1 \pm 3.5$ & -2.292 & 0.022 & -1.807 & 0.071 \\
\hline BPRS total score & $32.6 \pm 6.1$ & $29.7 \pm 6.5$ & $24 \pm 4.4$ & -2.481 & 0.013 & -4320 & $<0.0001 *$ \\
\hline CDSS total score & $5.1 \pm 4.8$ & $3.5 \pm 4.3$ & $2.5 \pm 3.7$ & -1.040 & 0.298 & -2.886 & 0.004 \\
\hline
\end{tabular}

a Wilcoxon test.

* Statistical significance after Bonferroni correction $(\alpha=0.002)$. 
Table 5

Cognitive functions at baseline (T0), at week 12 (T1), and at week 24 (T2) in patients receiving lamotrigine

\begin{tabular}{|c|c|c|c|c|c|c|c|}
\hline \multirow[b]{2}{*}{ Stroop test } & \multirow{2}{*}{$\begin{array}{l}\text { Baseline (T0) } \\
33.3 \pm 21\end{array}$} & \multirow{2}{*}{$\frac{\text { Week } 12(\mathrm{~T} 1)}{40.3 \pm 24.1}$} & \multirow{2}{*}{$\frac{\text { Week } 24 \text { (T2) }}{26.2 \pm 14.1}$} & \multicolumn{2}{|c|}{ Difference $^{\mathrm{a}} \mathrm{T} 0$ vs $\mathrm{T} 1$} & \multicolumn{2}{|c|}{ Difference $^{\mathrm{a}} \mathrm{T} 0$ vs $\mathrm{T} 2$} \\
\hline & & & & -2.945 & 0.003 & -2.177 & 0.029 \\
\hline Phonemic fluency & $28.7 \pm 7.3$ & $34.04 \pm 9.6$ & $30.4 \pm 8.7$ & -2.280 & 0.023 & -1.608 & 0.108 \\
\hline Semantic fluency & $39.1 \pm 10.7$ & $41.54 \pm 7.3$ & $41.9 \pm 7.3$ & -1.629 & 0.103 & -1.263 & 0.207 \\
\hline \multicolumn{8}{|l|}{$W C S T$} \\
\hline Perseverative errors & $33.5 \pm 23.3$ & $28.96 \pm 21.1$ & $30.2 \pm 16$ & -2.125 & 0.034 & -0.521 & 0.602 \\
\hline$T$ scores perseverative & $39.4 \pm 17.8$ & $42.54 \pm 17.8$ & $40 \pm 16.9$ & -1.613 & 0.107 & -0.317 & 0.752 \\
\hline Categories & $1.9 \pm 2.1$ & $1.92 \pm 2.3$ & $2.5 \pm 1.9$ & -0.060 & 0.952 & -2.147 & 0.032 \\
\hline
\end{tabular}

${ }^{\text {a }}$ Wilcoxon test.

task "perseverative errors" of WCST, observed at week 12 was not replicated at week 24 . When the Bonferroni correction was performed, no significant differences were observed (Table 5).

Plasma concentrations of clozapine $(m e a n \pm \mathrm{SD})$ did not change significantly during lamotrigine treatment (from $327 \pm 98 \mathrm{ng} / \mathrm{ml}$ at baseline to $336 \pm 106 \mathrm{ng} / \mathrm{ml}$ at week 24; plasma concentrations available from 22 patients) or with placebo (from $361 \pm 116 \mathrm{ng} / \mathrm{ml}$ at baseline to $345 \pm 100 \mathrm{ng} / \mathrm{ml}$ at week 24; plasma concentrations available from 20 patients). In the lamotrigine group, at week 24 plasma lamotrigine concentrations ranged from 1.7 to $5.3 \mu \mathrm{g} / \mathrm{ml}$ in 22 patients.

\section{Discussion}

The results obtained from the present study indicate that lamotrigine added to stable clozapine treatment showed a beneficial effect mainly on the negative and general psychopathological symptomatology in a sample of treatment-resistant schizophrenic patients. Lamotrigine was significantly more efficacious than placebo in reducing negative symptoms as measured by change on the SANS total scores and single domains alogia, affective flattening, avolition/apathy, anhedonia/asociality, and attention; the improvement was evident after 12 weeks of therapy and persisted until the end of the trial, at week 24. With regard to SAPS, lamotrigine was more effective than placebo in improving overall positive symptomatology, and principally the subscales hallucinations and thought disorder. The improvement in overall psychopathological state is highlighted by changes in BPRS scores during lamotrigine treatment. A reduction of affective symptomatology, as expressed by the reduction of CDSS total scores, was also observed. Conversely, in placebo treated patients no significant changes in the overall clinical state or in negative and positive symptoms were observed.
The reduction of general psychopathology during lamotrigine add-on treatment in schizophrenic patients has been documented in previous open trials (Dursun et al., 1999; Dursun and Deakin, 2001), brief reports (Kolivakis et al., 2004; Thomas et al., 2006), and randomized, double-blind, placebo-controlled trials (Tiihonen et al., 2003; Kremer et al., 2004). Besides the efficacy on general psychopathology, the results by Tiihonen et al. (2003) indicated that lamotrigine combined with clozapine significantly improved positive symptoms; similar findings emerged from the placebocontrolled trial by Kremer et al. (2004) which showed that positive symptoms and general psychopathology scores continued to decrease also during the 2-week fixed dose treatment with $400 \mathrm{mg} /$ day. Our results confirm the effect on general psychopathology and on positive symptoms, even if the reduction of positive symptoms is lower than expected. However, our study highlighted that lamotrigine combined with clozapine showed an evident therapeutic impact on negative symptoms, which, if replicated, would indicate lamotrigine as an effective therapeutic strategy for treatment-resistant schizophrenia with both dimensions of residual symptoms.

Cognitive functions did not significantly improve after lamotrigine treatment, with the exception of the semantic fluency at week 24 .

Consistent with previous findings (Tiihonen et al. 2003) lamotrigine was well-tolerated in long-term use, as only one patient dropped out for the development of a benign rash. The risk of developing a benign rash was estimated to be $8 \%$ (Calabrese et al., 2002), but it can be limited by adhering to the recommended slow initial rate of dose titration (Labiner, 2002). However, it must be kept in mind that its use is associated with the risk of exfoliative dermatitis which can affect on average 1 in 500 patients during long-term use (Hurley, 2002).

Adjunctive treatment of lamotrigine to an ongoing antipsychotic therapy can raise blood levels of antipsychotic drugs, as shown in a case report (Kossen et al., 
2001). This effect of the pharmacokinetic interactions has important clinical implications, as the rise in clozapine blood levels can cause marked side effects and an increase of toxicity (Szegedi et al., 1999). Moreover, it can be considered a potentially confusing element, as the improvement in clinical symptomatology reported after lamotrigine add-on therapy may result from an increase of clozapine plasma concentrations. As suggested by Dursun et al. (1999) on the basis of the observation that lamotrigine is metabolized in the liver by glucuronidation whereas clozapine is mainly metabolized by the isoenzyme 1A2 of the CPYP 450 system, any interaction between the two drugs was expected to be pharmacodynamic rather than kinetic. Consistent with this hypothesis, a recent study performed on a sample of 35 schizophrenic patients (Spina et al., 2006) showed that lamotrigine, at the dosages recommended for use as a mood stabilizer (up to $200 \mathrm{mg} /$ day) had little or no effect on the pharmacokinetics of the investigated antipsychotics. Moreover, lamotrigine did not alter the steady-state plasma concentrations of clozapine, risperidone and olanzapine and their active metabolites; only a modest, probably clinically insignificant, increase in the plasma concentration of olanzapine has been reported. The lack of significant kinetic interactions between lamotrigine and clozapine leads us to suppose that the therapeutic effect shown by lamotrigine on the clinical symptomatology of treatment-resistant schizophrenic patients is likely to result from a pharmacodynamic mechanism.

It has been suggested that the efficacy of lamotrigine augmentation on schizophrenic symptoms may involve the inhibition of excessive glutamate release resulting in overactive kainate and alpha-amino-3-hydroxy-5-methyl-4-isoxazolepropionate (AMPA) glutamate receptors (Tiihonen et al., 2003).

However, strong evidence indicates that lamotrigine is mainly effective in augmentation of clozapine treatment, leading to the hypothesis that lamotrigine may interact with the mechanism of action of clozapine (Dursun and Deakin, 2001).

It appears possible that the association of lamotrigine with clozapine may exert a synergistic action on the multiple receptor subtypes and on the neurotransmitter systems involved in the pathophysiology of schizophrenic symptoms. However, our results show certain limitations due to the small sample size, and the limited duration of the trial. Nevertheless, these findings provide evidence that lamotrigine augmentation of clozapine treatment is well-tolerated and may be proposed as an effective therapeutic strategy to improve outcome in treatment-resistant schizophrenia.

\section{Acknowledgements}

This study was supported by the Psychiatry Unit of the Department of Neurosciences, Psychiatric and Anaesthesiological Sciences, and by the Section of Pharmacology, Department of Clinical and Experimental Medicine and Pharmacology, University of Messina. The authors would also like to thank numerous scientific and practical collaborators, research participants and their families.

Conflict of interest statement

Authors declare that the manuscript is submitted on behalf of all authors. None of the material in this manuscript has been published previously in any form and none of the material is currently under consideration for publication elsewhere other than noted in the cover letter to the editor. Authors declare to have any financial and personal relationship with other people or organizations that could inappropriately influence this work.

\section{Contributors}

Prof. Rocco Zoccali designed the study and wrote the protocol.

Dr. Maria Rosaria Muscatello managed the literature searches and wrote the first draft of the manuscript. Dr. Antonio Bruno and Dr. Umberto Micò undertook the statystical analysis. Dr. Rosario Cambria managed clinical and neurocognitive assessment. Prof. Edoardo Spina and Prof. Mario Meduri supervised the Methods procedures, the various drafts and the final version of the manuscript. All authors contributed to and have approved the final manuscript.

\section{Role of funding source}

No sponsor provided funding for this study. The Psychiatry Unit of the Department of Neurosciences, Psychiatric and Anaesthesiological Sciences of the University of Messina provided the outpatients unit, the material for clinical and neuropsychological assessments, and electronic resources.

\section{References}

Addington, D., Addington, J., Maticka-Tyndale, E., 1993. Assessing depression in schizophrenia: the Calgary Depression Scale. Br. J. Psychiatry 163 (Suppl. 22), 39-44.

Anand, A., Charney, D.S., Oren, D.A., Berman, R.M., Hu, X.S., Cappiello, A., Krystal, J.H., 2000. Attenuation of neuropsychiatric effects of ketamine with lamotrigine: support for hyperglutamatergic effects of $N$-methyl-D-aspartate receptor antagonists. Arch. Gen. Psychiatry 57, 270-276.

Andreasen, N., 1983. Scale for the assessment of Negative Symptoms (SANS). University of Iowa, Iowa.

Andreasen, N., 1984. Scale for the assessment of Positive Symptoms (SAPS). University of Iowa, Iowa. 
Calabrese, J.R., Sullivan, J.R., Bowden, C.L., Suppes, T., Goldberg, G.F., Sachs, G.S., 2002. Rash in multicenter trials of lamotrigine in mood disorders: clinical relevance and management. J. Clin. Psychiatry 63, 1012-1019.

Chan, Y.C., Miller, K.M., Shaheen, N., Votolato, N.A., Hankins, M.B., 2005. Worsening of psychotic symptoms in schizophrenia with addition of lamotrigine: a case report. Schizophr. Res. 78 (2-3), 343-345

Dursun, S.M., Deakin, J.F.W., 2001. Augmenting antipsychotic treatment with lamotrigine or topiramate in patients with treatment-resistant schizophrenia: a naturalistic case-series outcome studies. J. Psychopharmacol. 15, 297-301.

Dursun, S.M., McIntosh, D., Milliken, H., 1999. Clozapine plus lamotrigine in treatment-resistant schizophrenia. Arch. Gen. Psychiatry 56, 950.

Gluck, M.R., Thomas, R.G., Davis, K.L., Haroutounian, V., 2002. Increased phosphate-activated glutaminase and glutamic acid decarboxylase activities in dorsolateral prefrontal cortex of aged schizophrenics: altered glutamate and GABA metabolism in schizophrenia. Am. J. Psychiatry 159, 1165-1173.

Goff, D.C., Coyle, J.T., 2001. The emerging role of glutamate in the pathophysiology and treatment of schizophrenia. Am. J. Psychiatry 158, 1367-1377.

Halberstadt, A.L., 1995. The phencyclidine-glutamate model of schizophrenia. Clin. Neuropharmacol. 18 (3), 237-249.

Haroutunian, V., Dracheva, S., Davis, K., 2003. Neurobiology of glutamatergic abnormalities in schizophrenia. Clin. Neurosci. Res. 3, 67-76.

Heaton, R., Chelune, G., Talley, J., Kay, G., Curtis, G., 1993. Wisconsin Card Sorting Test manual. Psychological Resources, Odessa, FL.

Holzer, L., Halfon, O., 2004. Normalization of impaired cognitive functions failed to improve clinical symptomatology in a schizophrenic patient. Eur. Psychiatr. 19, 384-386.

Hurley, S.C., 2002. Lamotrigine update and its use in mood disorders. Ann. Pharmacother. 36, 860-873.

Ishimaru, M., Kurumaji, A., Toro, M., 1994. Increases in strychnineinsensitive glycine binding sites in cerebral cortex of chronic schizophrenics: evidence for glutamate hypothesis. Biol. Psychiatry $35,84-89$.

Jentsch, J.D., Roth, R.H., 1999. The neuropsychopharmacology of phencyclidine: from NMDA receptor hypofunction to the dopamine hypothesis of schizophrenia. Neuropsychopharmacology 20, 201-225.

Kalyoncu, A., Mirsal, H., Pektaş, Ö., Ünsalan, N., Tan, D., Beyazyürek, M., 2005. Use of lamotrigine to augment clozapine in patients with resistant schizophrenia and comorbid alcohol dependence: a potent anti-craving effect? Psychopharmacology 19 (3), 301-305.

Kim, J.S., Kornhuber, H.H., Scmid-Burgk, W., Holzmuller, B., 1980. Low cerebrospinal glutamate in schizophrenic patients and a new hypothesis on schizophrenia. Neurosci. Lett. 20, 379-382.

Kolivakis, T.T., Beauclair, L., Margolese, H.C., Chouinard, G., 2004. Long-term lamotrigine adjunctive to antipsychotic monotherapy in schizophrenia: further evidence. Can. J. Psychiatry 49 (4), 280.

Kossen, M., Selten, J.P., Kahn, R.S., 2001. Elevated clozapine plasma level with lamotrigine. Am. J. Psychiatry 158, 1930.
Kremer, I., Vass, A., Gorelik, I., Bar, G., Blanaru, M., Javitt, D.C., Heresco-Levy, U., 2004. Placebo-controlled trial of lamotrigine added to conventional and atypical antipsychotics in schizophrenia. Biol. Psychiatry 56, 441-446.

Labiner, M., 2002. Lamotrigine and rash: scratching beneath the surface. J. Clin. Psychiatry 63, 1010-1011.

Large, C.H., Webster, E.L., Goff, D.C., 2005. The potential role of lamotrigine in schizophrenia. Psychopharmacology 181 (3), 415-436.

Munro, J., Matthiasson, P., Osborne, S., Travis, M., Purcell, S., Cobb, A.M., Launer, M., Beer, M.D., Kerwin, R., 2004. Amisulpride augmentation of clozapine: an open non-randomized study in patients with schizophrenia partially responsive to clozapine. Acta Psychiatr. Scand. 110, 292-298.

Overall, J.E., Gorham, D.R., 1962. The Brief Psychiatric Rating Scale. Psychol. Rep. 10, 799-812.

Premkumar, T.S., Pick, J., 2006. Lamotrigine for schizophrenia Cochrane Database of Systematic Reviews, Issue 4. Art. No.: CD005962. DOI: 10.1002/14651858.CD005962. pub2.

Saba, G., Dumortier, G., Kalalou, K., Benadhira, R., Degrassat, K., Glikman, J., Januel, D., 2002. Lamotrigine and clozapine combination in refractory schizophrenia: three cases. J. Neuropsychiatry Clin. Neurosci. 14, 86.

Simpson, M.D., Slater, P., Deakin, J.F., 1998. Comparison of glutamate and gamma-aminobutyric acid uptake binding sites in frontal and temporal lobes in schizophrenia. Biol. Psychiatry 44, 423-427.

Spina, E., D’Arrigo, C., Migliardi, G., Santoro, V., Muscatello, M.R., Micò, U., D’Amico, G., Perucca, E., 2006. Effect of adjunctive lamotrigine treatment on the plasma concentrations of clozapine, risperidone and olanzapine in patients with schizophrenia or bipolar disorder. Ther. Drug. Monit. 28 (5), 599-602.

Spreen, O., Benton, A.L., 1977. Neurosensory Center Comprehensive Examination For Aphasia (NCCEA). University of Victoria Neuropsychology Laboratory, Victoria.

Szegedi, A., Anghelescu, I., Wiesner, J., Schlegel, S., Weigmann, H., Hartter, S., Hiemke, C., Wetzel, H., 1999. Addition of low-dose fluvoxamine to low-dose clozapine monotherapy in schizophrenia: drug monitoring and tolerability data from a prospective clinical trial. Pharmacopsychiatry 32 (4), 148-153.

Tamminga, C.A., 1999. Glutamatergic aspects of schizophrenia. Br. J. Psychiatry 174, 12-15.

Tamminga, C.A., Holcomb, H.H., 2005. Phenotype of schizophrenia: a review and formulation. Mol. Psychiatry 10, 27-39.

Thomas, R., Howe, V., Foister, K., Keks, N., 2006. Adjunctive lamotrigine in treatment-resistant schizophrenia. Int. J. Psychopharmacology 9, 125-127.

Tiihonen, J., Hallikainen, T., Ryynänen, O.-P., Repo-Tiihonen, E., Kotilainen, I., Eronen, M., Toivonen, P., Wahlbeck, K., Putkonen, A., 2003. Lamotrigine in treatment-resistant schizophrenia: a randomized placebo-controlled crossover trial. Biol. Psychiatry 54, 1241-1248.

Trenerry, M.R., Crosson, B., DeBoe, J., Leber, W.R., 1989. Stroop Neuropsychological Screening Test. Psychological Assessment Resources, Inc., Odessa FL. 